\title{
LOS MORISCOS DEL REINO DE GRANADA DESPUÉS DE 1570
}

Nadie discutirá que 1570 es una fecha clave en la historia de los moriscos y del reino de Granada; la expulsión masiva de la que fueron víctimas, a raíz de su levantamiento, tiene largo alcance. Aun cuando generalmente se admite que algunos moriscos regresaron clandestinamente a su región de origen y que otros lograron permanecer ahí, se llegó a la conclusión de que el problema morisco había terminado en Andalucía oriental. Dos ejemplos ilustrarán el sentir general: "habían limpiado a Granada para atestar Castilla, sobre todo Castilla la Nueva" dice Fernand Braudel. Y para Miguel Ángel Ladero Quesada, cuya excelente obra abarca hasta el año de 1570, la Granada islámica "estaba muerta" a fines del siglo XVI; la leyenda había sustituido a la historia". Estas fórmulas son bellas pero merecen ser cuestionadas. ¿Los moriscos que se quedaron, o los que regresaron posteriormente, son realmente una pelusa que podemos barrer fácilmente? ¿Cuántos son? ¿Quiênes son? ¿Quế hacen? ¿Y no reflejan, sobre todo, la voluntad de una civilización minoritaria por rechazar la muerte?

Hasta la fecha, lo que sabemos acerca de ellos se limita a tres hechos básicos que es conveniente recordar. a) Unos 3500 moriscos granadinos fueron expulsados en 1584 . Henri Lapeyre describió muy bien este episodio, pero no nos hemos preguntado todavía sobre su significado ni sobre la condición de los expulsados². b) Por otro lado, las interpretaciones que Julio Caro Baroja y Darío Cabanelas dieron sobre el descubrimiento del pergamino de la torre Turpiana en Granada (1588) y de los libros plúmbeos del Sacromonte (1595) son muy convincentes ${ }^{3}$. Estos documentos

${ }^{1}$ F. BRAUDEL, La Méditerranée et le monde méditerranéen à la époque de Philippe II, Paris, 1966; M. A. LADERo QUEsada, Granada, historia de un país islámico (1232-1571). Madrid, 1969. [2ed., Madrid, 1980].

${ }^{2}$ H. LAPEYRE, Géographie de l'Espagne morisque, Paris, 1957, pp. 127-129.

${ }^{3}$ J. CARo BAROJA, Los moriscos del reino de Granada, Madrid, 1957, pp. 
reflejan una tentativa de sincretismo entre el cristianismo y el Islam, ideado por moriscos de renombre como Alonso del Castillo y Miguel de Luna. c) Por último, entre 1611 y 1614 se procedió a la expulsión definitiva de los moriscos hacia la Berbería o hacia la Provenza. Según Henri Lapeyre, de 1000 a 1500 granadinos fueron víctimas de esta expulsión. Pero, ¿podemos decir que esta vez el reino de Granada se vio definitivamente libre de los últimos sobrevivientes de la minoría perseguida? Es probable que un mejor conocimiento del medio morisco permitiría una mejor comprensión de estos tres sucesos y también una mejor comprensión de la supervivencia de esa minoría. Desde esta perspectiva intentaré, pues, plantear el problema de la comunidad morisca en el reino de Granada, entre 1570 y 1610 y más particularmente entre 1570 y 1585 .

Calcular el número de moriscos en esa época no es cosa fácil, no porque las autoridades no hayan intentado censarlos, sino porque es necesario tomar en cuenta los métodos adoptados y las condiciones particulares bajo las cuales se elaboraron los documentos. Tanto en 1578 como en 1580 , las mejores fechas para estudiar estos censos, se recurrió al clero secular que elaboró listas de moriscos parroquia por parroquia ${ }^{5}$. ¿Qué criterios emplearon los sacerdotes para definir a los moriscos? ¿No hubo falsas declaraciones favorecidas por una inmensa red de complicidades? Es posible que todos los que pretendían ser cristianos viejos hayan escapado al recuento. En 1573, setenta familias habian entablado un juicio para probar su origen cristiano, y su número aumentó en el curso de los años siguientes ${ }^{6}$. En fin, habría que agregar a todos aquellos que se escondieron, cuyo número era muy alto entre los esclavos. Las autoridades mismas no se dejaban engañar a este respecto, ya que insistían en la facilidad que había para los regresos clandestinos: "y según esto es de creer que después... se habrán vuelto y volverán de cada día otros muchos de las partes... donde están aloxados..." Por todos estos motivos, los datos de los censos deben considerarse como indicios mínimos. Así, el documento del 31 de enero de 1580 menciona 8698 personas. Ante la imposibilidad de mayor precisión, diré que en el reino de Granada había, hacia esa fecha, de.10 000 a 15000 moriscos. Si bien las limitaciones del documento de 1580 son evi-

211-212; D. CABANElAS, El morisco granadino, Granada, 1965.

${ }^{4}$ H. LAPEYRE, op. cit., pp. $181-182$

${ }^{5}$ Archivo General de Simancas, Cä́nara de Castilla, leg. 2176 y leg. 2178.

${ }^{6}$ Id., Cámara de Castilla, leg. 2179. 
dentes en cuanto al número de moriscos registrados, es bueno en muchos sentidos: la distribución geográfica de los moriscos, por ejemplo, es muy clara, como lo atestigua este cuadro:

Granada, su vega y valle

Motril y su partido

Alpujarras

Guadix y su tierra

Baza y su tierra

Almería

Río de Almería

Purchena y su tierra

Vera

Fiñana

Málaga

385
158
212
419
379
336
14
4
96
59

3851

158

212

419

379

336

14

42

96

59

1274
Vélez-Málaga

Ronda

Marbella

Loja

Alhama

Lugares de señorío del Obispado de Málaga

Marquesado del Cenete

Huéscar y su partido

Marquesado de los

Vêlez
385

289

145

330

77

71

106

238

222

La mayor parte de los cristianos nuevos se concentraban en grandes ciudades como Granada y Málaga, que reunían más del $50 \%$ del total. Zonas como Alpujarras, Almería, Purchena, Fiñana... en donde, antes de 1570 , los moriscos representaban la casi totalidad de la población están despobladas hacia esas fechas. La otra concentración relativa concierne a algunos señoríos cuyos dueños, la marquesa del Cenete, el duque de Alba (señor de Huéscar) y el marqués de los Vélez, no escatimaron esfuerzos para conservar una parte del campesinado.

Estos grandes señores, que gozaban del favor real, triunfaron en sus esfuerzos. En estas condiciones, la distribución geográfica de los moriscos no se parece en nada a la de la primera mitad del siglo, que revelaba una población rural en su mayor parte, concentrada sobre todo al este del reino de Granada.

También es posible ver a la población morisca desde un punto de vista sociológico, aspecto en el que contamos con más certidumbre. Retomemos el documento de 1580 a partir del segundo cuadro que nos presenta:

Seises, conocedores, regadores oficiales. . . 328

Familia destos 1017

Moriscos mayores de 70 años $\quad 31$

Libres sin horden mayores de 21 años $\quad 311$

Familia destos $\quad 210$

Moriscas libres que viven de por si $\quad 1166$

Esclavos mayores de 21 años $\quad 74$

Esclavas 2593

Esclavos menores de 21 años $\quad 472$

Esclavas $\quad 645$

Moriscos menores de 21 años en administración $\quad 911$

Moriscas 904

Berberiscos varones $\quad 20$

$\begin{array}{ll}\text { Berberiscas } & 19\end{array}$ 
Varios de los términos empleados aquí merecen un comentario. Los seis eran, antes de 1570 , en el seno de una parroquia, los representantes encargados de controlar el reparto de la farda, impuesto pagado únicamente por los moriscos. Elegidos en cada aldea por un período de seis años, los seises eran seis: dos delegados por los ricos, dos por los de clase media y dos por los pobres ${ }^{7}$. Como habían establecido un censo anual, conocían muy bien a la población de la que eran procuradores. Por ello se comprende fácilmente que muchos hayan obtenido el derecho de permanecer en el lugar. El conocedor no difiere en nada del seis, salvo por el hecho de que el término no implica ninguna función o delegación particular. Los escribientes ponen, en la misma categoría que los seises y los conocedores, toda una serie de oficios, como el primero que citan, que se relaciona con las técnicas del riego. Conocemos en detalle esta serie, que se refiere casi exclusivamente a Granada. Aparecen 17 personas dedicadas al tinte de la seda, 12 regadores, 11 carpinteros, 6 herreros, 6 escofinadores de azúcar, 4 caldereros, 3 herradores y 20 moriscos de profesiones diversas: maestro de la gineta, ensalmador, jardinero, alfarero. . ${ }^{8} \mathrm{El}$ denominador común de todos estos indivi duos, desde el seis hasta el alfarero, es, por un lado, la libertad y la posesión y, por otro, el hecho de ser poseedor de una cédula real que aseguraba su estancia en el reino de Granada.

El término "en administración" intriga. Los autores del cuadro tuvieron buen cuidado de establecer una distinción entre un buen número de moriscos menores de 21 años y los esclavos de la misma edad. Estos últimos eran, en realidad, niños huérfanos libres que dependían de una persona, a la cual habían sido confiados en virtud de un contrato firmado ante notario ${ }^{9}$. Otros textos emplean como equivalentes de "en administración", las expresiones "encomendación" o "soldada".

Por lo que toca a los berberiscos, que no eran moriscos, sino originarios del Âfrica del Norte, tenían derecho de ciudadanía en el reino de Granada. Por esto mismo, algunos moriscos no dudaron en utilizar esta licencia para hacerse pasar por berberiscos y, de este modo, permanecer en Granada.

La distribución social de los moriscos tal y como aparece en 1580 es, en gran parte, consecuencia del modo como se aplicó el

${ }^{7}$ K. Garrad, The original Memorial of Don Francisco Núñez Muley, Atlanta, 1954.

${ }^{8}$ Archivo General de Simancas, Cámara de Castilla, leg. 2180.

${ }^{9} \mathrm{~N}$. CABRILlaNA, "Almería en el siglo XVI: moriscos encomendados", $R A B M, 73$ (1975), 401-468. 
decreto de expulsión en 1570. En teoría, todos los moriscos estaban implicados en este decreto. En la práctica, las excepciones fueron múltiples y se aplicaron, sobre todo, a los esclavos y a las mujeres. En 1580, 5599 de los 8701 moriscos censados son dependientes, lo que viene a constituir cerca de los dos tercios, incluyendo a los que se encuentran "en administración". No hay duda - pero aquí los datos no son tan claros - de que la proporción de dos tercios de mujeres por un tercio de hombres está muy cercana a la realidad. Las mujeres son más numerosas debido a que dos categorías, la de esclavas adultas y mujeres libres, suman un total de 1166 y es posible que fueran viudas en su mayoría.

A estas dos características fundamentales hay que agregar la pobreza. Resulta inútil hacer comentarios sobre los bienes de los dependientes. Los únicos que poseían algunos, es decir, aquellos que estaban colocados en administración, no tenían más de una veintena de ducados al ser liberados de la tutela. Las viudas, que vivían en la indigencia más completa, trabajaban seguramente en la industria de la seda, que era objeto de todas las atenciones y cuidados de la administración. La mano de obra barata que representaban estas mujeres aseguraba su continuidad.

Finalmente, entre los que obtuvieron la autorización real de permanecer en Granada y cuya fortuna conocemos, más de la mitad, es decir 178 sobre 307 (58\%), no poseían bienes, según los registros. Incluso el número podría elevarse al $61 \%$, aun si no tomamos en cuenta a los seises y a los conocedores de Granada, porque no había muchos pobres entre los titulares de la cédula real. Los que poseen bienes rara vez son "pequeños", en el sentido que da a esta palabra Fernand Braudel. La mayoría alcanza el nivel de "razonables" y una importante minoría, el de los ricos. De los 128 jefes de familia "poseedores", únicamente 22 no rebasan el límite de los 100 ducados; 53 se sitúan entre 120 y 350 , y 53 en 400 y más. Los más ricos eran: el tintorero granadino Iñ̄igo Rodríguez, que poseía 2000 ducados, y el seis de Caniles, Juan de Ronda el Haquin, que tenía 1600.

Geográficamente aparecen matices notables que probablemente traicionan la variedad de calidad de los terruños, así como las consecuencias de la guerra de 1568-1570; esto último, según que nos encontremos en un pueblo que no haya sido atacado o en uno que haya padecido los rigores de las batallas. De los 128 privilegiados, 26 pertenecen a la tierra de Baza (Benamaurel, Cullar, Caniles, Zujar). En estos 4 pueblos, frente a 26 que tienen fortuna, sólo figuran 4 sin bienes. El otro polo de la riqueza se encuentra en la misma Granada, entre los seises y los conocedo- 
res; de éstos, 24 sobre 35 poseen bienes. Es importante subrayar que tanto los seises de la tierra de Baza como los de Granada tienen, cada uno, un mínimo de 150 ducados, y constituyen la mitad de los "razonables" y ricos.

Existen otros tres sectores distintos de los anteriores. En primer lugar, el de los artesanos granadinos, en donde las grandes fortunas de los tintoreros o de los carpinteros van a la par de la miseria de la mayoría. En total, de 90 jefes de familia, 19 poseen riquezas. La Vega de Granada es un pequeño mundo equilibrado: 28 que tienen bienes, 29 no, y es de notar, entre los primeros, que la cantidad de valores que poseen fluctúa entre 20 y $300 \mathrm{du}$ cados. Por último tenemos a los 13 seises del obispado de Málaga, de los cuales únicamente cuatro poseen bienes.

Por encima de las tres categorías - dependientes, trabajadores pobres, trabajadores acomodados o ricos - que hasta aquí he definido, figuran las familias "colaboradoras". Descendientes, generalmente, de los grandes linajes del reino nazarí, estas familias prestaron su apoyo a los soberanos españoles desde la Reconquista. Al señalar las excepciones a la drástica medida de la expulsión, las autoridades supieron reconocer la ayuda que se les había brindado. Esas 32 familias deben ser mencionadas porque el papel que desempeñó cada una de ellas es digno de estudio.

GRANADA: Hijos de'don Gonzalo el Zegri, Don Francisco el Zegri, Don Alonso Vanegas, Don Gerónimo Vanegas, Don Alonso Vanegas de Alarcón, Pedro Vanegas, Gerónimo de Palacios, Miguel de Palacios, el doctor Pedro de Pinar Fustero, Lope Fustero escrivano, Gavriel Tristán Fustero escrivano público, Hernando Arias Fustero, García de la Rua escrivano, Alonso Hernández Gavano escrivano, Luis Méndez receptor de la audiencia, el licenciado Castillo médico, Alvaro de León, los sobrinos del padre Albotodo, Don Fernando de Fez Muley.

Guadix: Don Alonso Vanegas de Almería, Fernando de Belbis, El maestrescuela de Almería y sus hermanos y sobrinos, Alonso de Montanos.

VÉLEZ-MÁlAGA: Bernardino de la Reina regidor, Juan de la Reina su hermano jurado, Pedro Gómez regidor (suegro de Juan de la Reina).

MÁlAgA: Don Juan de Málaga regidor, Juan Esteban ${ }^{10}$.

Estas 32 familias no representan todos los colaboracionistas moriscos, ya que en la lista no se mencionan aquéllos, menos co-

${ }^{10}$ Esta lista, al igual que todos los datos referentes a las fortunas, proviene del documento citado en la nota 8. 
nocidos, provenientes del campo en su gran mayoría, que cumplieron alguna tarea oscura. Pero esas familias constituyen la parte visible, casi oficial y, por lo tanto, compleja. Ocupan el primer lugar las grandes familias, Zegri, Vanegas, Palacios, León, Fez Muley, Málaga, cuyos miembros ocuparon numerosos cargos públicos. En seguida vienen los fieles miembros de la administración: magistrados o notarios. Finalmente, aquéllos cuya actividad fue ejemplar en el plano religioso o diplomático: los Albotodo, Alonso Castillo, la familia Marín. Todos ellos tienen el prestigio que les confirió su linaje, su renombre o el favor del Estado. Este grupo, que comprende entre 150 y 200 personas, constituye un medio homogéneo dados los lazos de sangre que existen entre muchos de sus miembros (seis linajes, los de Zegri, Vanegas, Palacios, Fustero, López Abenaxara, de la Reina, agrupan a 19 de estas 32 familias) y dadas también las relaciones de vecindad, ya que la mayor parte de estos moriscos habitan en la parroquia de San Miguel de Granada.

Algunos de estos notables, como Alonso del Castillo por ejemplo, son bien conocidos ${ }^{11}$, pero la mayoría merecería salir del anonimato. Aquí tomaré solamente tres casos. En primer lugar a Francisco el Zegri, cuya larga vida está jalonada de leales servicios prestados a la corona de España. En 1525 tomó parte en el desafortunado ataque al Peñón de Vélez de la Gomera, en las costas africanas, en donde cayó prisionero, y permaneció en cautiverio por espacio de quince meses ${ }^{12}$. Su rescate se obtuvo mediante el pago de 1800 ducados y la liberación de un musulmán que se encontraba en manos del rey de Portugal. Después de una expedición a Tánger, acude a la de Túnez como capitán de caballería del duque de Sesa. En esta última expedición se cubre de gloria al apoderarse de piezas de artillería enemigas. En 1543 , como miembro de la flota que se encontraba bajo el mando de Alvaro Bazán, se apodera de la nave capitana francesa a la altura del cabo Finisterre ${ }^{13}$. Finalmente, en 1569 y 1570 , pertenece al ejército de don Juan de Austria en las Alpujarras.

Paralela a su carrera militar ha desarrollado otra, política y administrativa en el reino de Granada. En 1561 vive en la parroquia de San Juan de los Reyes, en la Alcazaba Cadima, rodeado

${ }^{11} \mathrm{D}$. Cabanelas, op. cit.

${ }^{12}$ El rey participó en el rescate por medio de una donación de 1000 ducados. Archivo General de Simancas, Cámara Cédulas, libro 255, fol. 346 v.

${ }^{13}$ Francisco el Zegri obtiene también la cédula real para portar armas. Archivo General de Simancas, Cámara Cédulas, libro 256, fol. 142, texto del $1 / \mathrm{XII} / 1550$. 
de su mujer María, de su hermano y de cuatro criados, y es responsable del reparto de la farda durante largo tiempo ${ }^{14}$. Como veinticuatro de Granada desde 1536 hasta su muerte, en 1578 desempeña un papel predominante en el consejo municipal del cual es decano por muchos años. Como tal, es el primero en emitir su voto sobre cualquier tipo de asunto convirtiéndose, de este modo, en el agente de la política real ${ }^{15}$. Este celo le convierte en objeto del odio de los moriscos. En el transcurso de la guerra son quemadas sus propiedades rurales, con lo que Francisco el Zegri se encuentra prácticamente en la ruina al final del conflicto ${ }^{16}$. En 1574 Pedro de Deza, presidente del Consejo de Población, interviene ante el rey en su favor recordándole que Carlos $\mathrm{V}$ había otorgado a Francisco el Zegri, en 1543, una renta anual de 100 ducados deducida del producto de la farda, la que se le dejó de pagar en $1568^{17}$. El Zegri recibe entonces un juro de 100 ducados, que, a su muerte, es transmitido, de acuerdo con los deseos de la ciudad de Granada, a Juan, su hijo de siete años.

El otro caso es el de la familia Albotodo. No mencionaremos el caso del jesuíta Juan Albotodo que ha sido ya bien estudiado, sino más bien daremos algunos datos sobre la familia en su conjunto $^{18}$. El que fue a partir del año 1550, el evangelizador más activo entre sus antiguos correligionarios, descendía de una familia de herreros y caldereros que, desde muy temprana época, había abrazado la religión católica. Dos miembros de esta familia, habitantes de Granada, obtuvieron en 1511 el privilegio, raro entre los moriscos, de portar armas. Uno de ellos, Alonso - el padre de Juan se llamaba Alonso, ¿será el mismo? - lo obtuvo el 24 de mayo y el otro, Bartolomé, el 6 de octubre ${ }^{19}$. Para aquel entonces ya habían sido admitidos como cristianos verdaderos.

Juan Albotodo tenía cinco hermanos: Alonso, Bernabé, Miguel, Ángela y María. Casi todos ellos vivían en 1561 en la parroquia de San Miguel de Granada. Alonso vivía en una casa con su esposa y un sirviente, Bernabé en la otra, con la que tal vez era su esposa, y el padre Juan vivía en otra casa, en compañía de cinco

${ }^{14}$ Archivo General de Simancas, Cámara de Castilla, leg. 2150.

${ }^{15}$ Ibid., leg. 2178, texto del $24 / \mathrm{III} / 1574$.

${ }^{16} \mathrm{Ibid}$., leg. 2181, texto del $13 / \mathrm{X} / 1578$.

${ }^{17}$ Ibid., leg. 2172.

${ }^{18}$ A. MARIN OCETE, El arzobispo Pedro Guerrero, t. 2, pp. $411 \cdot 412,423$ 424; A. GARRIDo ARANDA, "Papel de la iglesia de Granada en la asimilación de la sociedad morisca", Anuario de Historia Moderna y Contemporánea, 1975-1976, pp. 92-93 y 101-103.

${ }^{19}$ Archivo General de Simancas, Cámara de Cédulas, libro 255. 
personas entre las que se encontraban dos esclavos negros ${ }^{20}$. Alonso tuvo un fin trágico: encarcelado junto con varias decenas de moriscos, en abril de 1568 , por las autoridades que querían evitar un levantamiento, murió, al igual que otras ciento diez personas, el 17 de abril de 1569. Su cuñado Felipe Vanegas, marido de María de la Cruz, tuvo el mismo fin ${ }^{21}$. Bernabé murió, al parecer, de muerte natural antes de 1570 . Su viuda, que contrajo segundas nupcias con un morisco, continuó viviendo en su casa de San Miguel, en donde la encontramos en 1572. Al año siguiente, los miembros sobrevivientes de esta familia, tres parejas y sus hijos, en total dieciséis personas, pidieron la restitución de los bienes que les habían sido confiscados: casas, tierras laborables y viñedos, valuados entre 1300 y 1600 ducados. Aquí también intervino en su favor Pedro de Deza y todo hace pensar que triunfó en su propósito ${ }^{22}$.

El último caso al que aludiré es ajeno a la lista de los 32 jefes de familia citados anteriormente. Se trata de Íñigo Rodríguez. Con él veremos cómo los más encopetados de los seises, conocedores y artesanos que permanecieron en el reino de Granada difieren poco, en realidad, de las grandes familias que acabo de mencionar. La trayectoria es idéntica. Iñigo Rodríguez el Gamad o Gamudi, tintorero que poseía una fortuna de alrededor de 2000 ducados, es, en realidad, el hijo de Diego, a quien los Reyes Católicos pidieron en 1501 que redactara las famosas ordenanzas del arte de la seda ${ }^{2 s}$. Diego Rodríguez ejerció, durante más de cincuenta años, el oficio de alamin, inspector de tintoreros, fue eximido de los impuestos que pesaban sobre los moriscos, obtuvo, en virtud de una cédula otorgada el 26 de marzo de 1518 y confirmada el 26 de octubre de 1526, el derecho de portar armas, y recibió, a partir de 1526, una renta anual de 6 ducados $^{24}$.

Íñigo permaneció fiel a la línea de conducta de su padre: militó al lado de los cristianos durante la guerra de 1568-1570, y dedicó un fondo para el monasterio de los Jerónimos de Granada ${ }^{25}$.

${ }^{20}$ Archivo General de Simancas, Cámara de Castilla, leg, 2150.

${ }^{21}$ L. DEL MÁRMOL CARVAJAL, Historia de la rebelión y castigo de los moriscos de Granada, t. 21, B.A.E., Madrid, 1946, pp. 176 y 252.

${ }^{22}$ Archivo General de Simancas, Cámara de Castilla, leg. 2174; documento del $30 / \mathrm{IV} / 1573$.

${ }^{23}$ M. GARZÓN PAREJA, La induistria sedera en Esṕaña; el arté de la seda de Granada, Granada, 1971, pp. 389 ss.

${ }^{24}$ Archivo General de Simancas, Cámara de Cédulas, libro 255, fols. 375 v y $382 \mathrm{v}$.

${ }^{25}$ Archivo General de Simancas, Cámara de Castilla, leg. 2180. 
Con los moriscos colaboracionistas tenemos el cuarto elemento, y no por ello el menos importante, de la comunidad que permanecía en Granada después de 1570. Los detalles presentados en párrafos anteriores son válidos, esencialmente, para los años 1577-1580, fechas para las cuales disponemos de cuadros sinópticos. Aun cuando pudieran variar los números según los años, la composición de la comunidad que hemos establecido es válida, al menos, de 1570 a 1584 , y podemos decir que posteriormente no sufre modificaciones notables.

El quinto elemento, los monfíes, desapareció hacia 1577. Desde el final de la guerra, en 1570, muchos hombres huyeron a los montes, y organizaron bandas que sembraron el terror entre la población. Se hicieron muchos intentos de poner fin a sus fechorías, pero fueron necesarios muchos años para lograrlo. En 1577 se sometieron los últimoṣ jefes: Juan Esvile, Marcos el Meliche..., sobrevivientes de una aventura que contó con centena res de hombres en $1572-1573^{26}$.

Una vez descrita la comunidad morisca, es posible entrever cuál será su porvenir y, sobre todo, comprender mejor el alcance de la expulsión parcial de 1584, de los libros plúmbeos del Sacromonte y de la expulsión de 1610 y 1611 . Aquí es importante conocer el juego de las relaciones entre la comunidad morisca fragmentada, la comunidad cristiana y el estado. Este último vive obsesionado por la amenaza que representan los moriscos, y no tiene la mejor intención de tomar en cuenta los casos especiales. Cualquier morisco - excepto los colaboracionistas - es considerado sospechoso y, por lo tanto, puede ser víctima, en cualquier momento, de la expulsión. A partir de 1571, se acusa a los moriscos que permanecieron en el reino de Granada, sobre todo a los esclavos, de complicidad con los monfíes o de engrosar sus filas. En numerosas ocasiones, entre 1571 y 1573, se intenta eliminarlos de toda Andalucía. La alerta más seria tiene lugar en noviembre de $1573^{27}$. Los seises, que, a primera vista, parecerían más protegidos, son amenazados por un bando del 3 de noviembre de 1577. Pero más importante todavía son las tentativas globales llevadas a cabo para limpiar el reino de Granada de su población morisca. Los únicos que se salvaron de estas medidas fueron los poseedores de la famosa cédula real. El 6 de mayo de 1576 , el 21 de julio de 1578 , el 4 de abril de 1579 y el 26 de enero

${ }^{26} \mathrm{~B}$. VINCENT, "Les bandit morisques en Andalousie au xvi" siècle", Revue d'Historie Moderne et Contemporaine, 1974, pp. 389-400.

${ }^{27}$ Archivo General de Simancas, Cámara de Castilla, leg. 2174. 
de 1581 se promulgan edictos que ordenan la expulsión de moriscos libres y esclavos. Los únicos casos de duda conciernen a los menores de 14 años y a los mayores de 70 . La repetida publicación de estos edictos es prueba de que su aplicación no satisfacía a las autoridades, aunque su efecto no fue nulo, como lo demuestran algunas listas que poseemos. Es así que 74 moriscos provenientes de Almería, 32 de Purchena y Motril, 11 originarios de Almuñecar y Motril y 51 del marquesado de los Vélez llegan a Toledo, sucesivamente, el 22 de noviembre de 1571, el 22 de febrero, el 3 de agosto de 1573 y el 26 de abril de 1574 .

La explicación de los fracasos sucesivos de las medidas gubernamentales es bastante simple. La casi totalidad de los moriscos de Andalucía oriental sólo pudieron permanecer ahí gracias al consentimiento de los cristianos. La compenetración entre estas dos comunidades iniciada a principios del siglo XVI y más sólida en el medio urbano de lo que se ha venido afirmando hasta el presente, había llevado a los cristianos a adoptar algunos elementos de la civilización contraria y también a no considerar la comunidad morisca como un bloque. Los granadinos, especialmente, eran propensos a establecer una distinción entre moriscos irreductibles y moriscos asimilados. ¿Para qué expulsar a los mejores ya que no formaban un grupo demasiado numeroso? Importaba a los habitantes de las ciudades del reino de Granada no sentirse amenazados físicamente por "el otro"; una vez logrado esto, su presencia era aceptada y, más aún, deseada.

Hoy en día se considera que una comunidad tolera a una minoría siempre y cuando sus miembros no sobrepasen el $10 \%$ de la población total, ya que, si rebasa este límite, sobreviene el rechazo de unos hacia otros. Así sucedió en Andalucía oriental. Después de 1570 los moriscos constituían menos de la décima parte de la población y, circunstancia favorable a su permanencia, no trataron jamás de agruparse en alguna morería, contrariamente a lo que sucedió en la mayor parte de las ciudades castellanas. A estas alturas, la cohesión, la solidaridad ya no existían. Un último elemento les favoreció: a lo largo del siglo XVI, se sometió a los moriscos a los trabajos más duros y pesados. En Andalucía oriental, a partir de 1570 , esto, la codicia en el trabajo que se reprobaba a los moriscos, se convierte en ventaja para los cristianos, ya que con el servicio de los moriscos podían abstenerse de tareas que no podían o no querían hacer. De este modo se reunían las condiciones necesarias para la permanencia de una gran comunidad dispersa: no sólo no era peligrosa sino que era útil. 
Las expulsiones limitadas de los años 1573 y 1574 anuncian aquéllas, más importantes, de 1584 . El texto del 26 de enero de 1581 tuvo un alcance mayor que los precedentes. No obstante, fueron necesarios otros tres años para que los agentes de Felipe II lograran su objetivo, y no porque técnicamente las operaciones fueran complicadas, porque desde los años $1569-1570$ ya se conocen los procedimientos a seguir. Por otra parte, los sacerdotes habían establecido en cada parroquia las listas de los moriscos residentes. Con todo, fue necesario además vencer la resistencia local: sin caricaturizar demasiado, podemos afirmar que la única persona favorable a la expulsión en una ciudad era el corredor. Arévalo de Zuazo, por ejemplo, confiesa el $1^{\circ}$ de agosto de 1581: "en lo de los moriscos libres y cautivos que ay en esta ciudad y rreyno se executan los bandos con mucha floxedad..."

Los cristianos del reiro de Granada no se contentan con una oposición pasiva a las disposiciones reales, sino que transmiten sus opiniones a los despachos de la corte, opiniones que reflejan, en última instancia, en vísperas de la expulsión de enero de 1584, una verdadera campaña contraria a la expulsión. El obispo de Guadix y el arzobispo de Granada, las ciudades de Loja, Guadix, Antequera y Alcalá la Real manifiestan al rey su desacuerdo, y el de sus feligreses y administrados, por medio de cartas dirigidas, sucesivamente, el 9, el 14, el 15, el 17 y el 21 de enero de ese año.

Todos ellos emplean los mismos argumentos: el carácter indispensable de la mano de obra morisca y la aculturación casi completa de la mayoría de los moriscos afect ados por el edicto real. Este sentimiento, expresado de este modo en 1584, se había propagado ya desde 1574 según lo reflejan las elocuentes palabras del corregidor de Alcalá la Real a Pedro de Deza: "le querían apedrear los vezinos porque lo sintieron más que si les sacaran sus mismos hijos tanto es el amor que tienen con ellos y el aborrecimiento con quien haze executar lo que se les manda..." 28

Todas estas presiones dieron por resultado dos cosas: retardar al máximo la ejecución de tal medida y reducir notoriamente el número de víctimas. Los siete comisarios encargados de la expulsión cumplieron con su misión a partir de la segunda quincena de enero de 1584. En el curso del primer trimestre de ese año, de 3000 a 3500 personas fueron escoltadas hacia Castilla la Vieja: Sepúlveda, Aranda, Olmedo, Medina del Campo y Ciudad Rodrigo; hacia Castilla la Nueva: Toledo, Guadalajara, Almadén; y hacia Extremadura: Trujillo. Toledo, punto de convergencia de

${ }^{28}$ Ibid., leg. 2175, texto del 11 de noviembre de 1574 . 
numerosos caminos, sirvió de punto de enlace. Almadén, por su parte, acogió a más de doscientos moriscos destinados a trabajar en las minas de mercurio. En este último caso, las autoridades asintieron a las peticiones apremiantes de los' Fúcares.

Estas expediciones no son más que la repetición de las de 1570 , excepto por una diferencia esencial. Unas y otras constituyen un genocidio, pero mientras que podemos comprender las razones del Estado en 1570, éstas nos parecen irrisorias en 1584. Las víctimas de la expulsión, todas gente pobre, no representaban peligro alguno. Uno de los comisarios, Juan de Ávila, dice que entre las 800 personas que conduce hay un centenar de mujeres que van descalzas. Otro de ellos, Riano, precisa que las 217 personas que conduce son niños y jóvenes pobres de 12 a 20 años. Y un tercero, Núñez de Valdivia, describe la pobreza de la mayor parte de los 646 moriscos que se encuentran a su cargo, y subraya que los que están en "administración" van generalmente desnudos.

Estos testimonios, y muchos más, muestran perfectamente qué tipo de gentes son los nuevos expulsados. En parte se trata, sin duda alguna, de los que regresaron clandestinamente al reino de Granada, pero en su mayoría son dependientes. A los colaboracionistas no se les hostigó nunca. Las autoridades locales, con su tenacidad, salvaron casi a todos aquéllos cuyo trabajo se consideraba indispensable, es decir, además de los titulares de cédulas reales, a las mujeres libres. A 786 de ellas se las preservó de la expulsión con el fin de perpetuar la industria de la seda, particularmente el hilado, en todo el reino. Los dependientes no tenían, a los ojos de las municipalidades, igual importancia económica. La mayor parte de ellos eran sirvientes, y únicamente sus amos podían intervenir en su favor, y, en efecto, algunos de ellos, los más poderosos, lograron ser escuchados. Por lo menos 262 mujeres en administración pudieron quedarse ${ }^{29}$, pero todos los demás, en administración o esclavos, se fueron. En octubre de 1583, 34 esclavos del este del reino de Granada habían sido conducidos fuera de los límites del reino, sobre todo hacia la región de $\mathrm{Murcia}^{30}$, y todavía 404 en agosto de 1585 en dirección de Llerena, Mérida y Cáceres.

He aquí el triste epílogo de cinco años de esfuerzos desmedidos que sólo condujeron a desarraigar todavía más a unos huérfanos totalmente aculturados. Después de haberles quitado a sus familias quince años antes, ahora se les separaba a la vez de su tierra y de lo que podía considerarse su familia adoptiva. Eran

\footnotetext{
${ }^{29}$ Archivo General de Simancas, Cámara de Castilla, leg. 2186.

${ }^{30}$ Ibid., leg. 2185, documento del 4 de octubre de 1583 .
} 
gente totalmente asimilada porque no tenía ya nada en común con los moriscos de la primera expulsión. A finales de 1585, el corregidor de Trujillo constata que de los 166 moriscos que el comisario Riano le había entregado el año anterior, únicamente 33 permanecían en la ciudad. El resto (126) había huido tal vez en búsqueda desesperada de su lugar de origen, tal vez para mostrar su rechazo al destino que le había sido deparado.

Los sobrevivientes no volvieron a tener otra alarma hasta 1610. Los varios millares de moriscos que se habían quedado vieron aumentar su número debido a los regresos clandestinos que no cesaron nunca. La minoría se vio entonces tentada por dos cosas que haríamos mal en juzgar contradictorias: por un lado, fundirse con la mayoría, empleando para ello diversos medios entre los que figuraba, en primer lugar, el matrimonio mixto; y por otro lado, conservar lo esencial de su identidad. El episodio de los libros plúmbeos del Sacromonte, descubiertos en 1595, debe colocarse en este contexto. Como ya quedó establecido, los 19 libros en los que se admite, por un lado, la infalibilidad del Papa y la Inmaculada Concepción y, por otro lado, que Jesucristo no era hijo de Dios, responden a una tentativa de sincretismo, algunos de cuyos autores son conocidos: tal es el caso de los moriscos Alonso del Castillo y Miguel de Luna. Cómo no imaginar que a través de estos hombres se expresa toda la comunidad morisca del reino de Granada.

Este grupo, dominado más que nunca por los colaboracionistas, se caracteriza por poseer dos culturas y por estar consciente de que una de ellas, la musulmana, se está empobreciendo. En su afán desesperado por mantenerla viva, tratan, por la vía del sincretismo, de asegurar su supervivencia en el plano religioso. Sin embargo, pronto se revela la superchería y la tentativa fracasa; ya no queda a los moriscos más recurso que fundirse con la mayoría. El hecho es que el número de moriscos condenados por la Inquisición de Granada es relativamente elevado hasta 1584 y 1585. Después desciende a tal punto que ya no se designa a los moriscos como moriscos sino como blasfemos, bígamos, etc., y a finales del siglo XVI pululan los juicios contra los moriscos que pretenden descender de un cristiano. En algunos casos, los moriscos inventan estas filiaciones en provecho propio, pero en otros se trata de filiaciones reales, lo que explica por qué, en el momento de la expulsión final, el número de graradinos embarcados fue escaso. La mayoría pudo permanecer en Granada gracias a la complicidad de la población local. 
A través del ejemplo granadino, vemos que los moriscos que permanecieron en España después de 1610 y 1614 no constituían en modo alguno una cantidad desdeñable, como se había admitido generalmente hasta ahora. Gracias a ello los moriscos prolongaron, al menos en el reino de Granada, la presencia de una minoría, asegurando asî las transferencias culturales que un siglo de coexistencia, aun en circunstancias difíciles, no pudo dejar de provocar.

Casa de Velázquez. 